\title{
Common Fixed Point Theorems in Fuzzy Metric Spaces
}

\author{
Raghu Nandan Patel ${ }^{1}$, Manoj Kumar Tiwari ${ }^{2}$ \\ ${ }^{1}$ Government Mukut Dhar Pandey College, Katghora Distt. -Korba, Chhattisgarh, India \\ ${ }^{2}$ Government Girls Polytechnic , Bilaspur, Chhattisgarh, India
}

\begin{abstract}
In this paper we used the concept of compatible of type $(P)$ in fuzzy metric spaces. Our result generalize earlier results due to Vasuki [18], Chugh and Kumar [3] and others.
\end{abstract}

2000 Mathematics Subject Classification: 54H25, 47H10.

Keywords: fuzzy metric space, fixed point, compatible mappings of type $(P)$.

\section{Introduction}

The notion of fuzzy set was introduced by Zadeh [20] which laid the foundation of fuzzy mathematics. Consequently the last three decades were very productive for fuzzy mathematics and the recent literature has observed the fuzzification in almost every direction of mathematics such as arithmetic, topology, graph theory, probability theory, logic etc. Deng[ 4], Erceg [5], Kaleva and Seikkala [11] and Kramosil and Michalek [12] have introduced the concept of fuzzy metric spaces in various ways. George and Veeramani [8] modified the concept of fuzzy metric spaces introduced by Kramosil and Michalek [12] and defined Hausdorff topology of metric spaces which is later proved to be metrizable. They also showed that every metric induces a fuzzy metric and by now there exists considerable literature on this topic. Recently, Chugh and Kumar [3] proved a Pant [13, Theorem 1] type theorem for two pairs of R-weakly commuting mappings satisfying a Boyd and Wong [1] type contraction condition which in turn, generalizes a fixed point theorem of Vasuki [18, Theorem 2].

The purpose of this paper is to improve the main theorem of Vasuki [18] along with Theorem A (due to Chugh and Kumar [3]) besides adopting R-weak commutativity of type (Af ), type $(A g)$ to fuzzy setting and to introduce R-weak commutativity of type $(P)$ which are to be used to prove our results in this paper.

\section{Preliminaries}

Definition 2.2. A fuzzy set $A$ in $X$ is a function with domain $\mathrm{X}$ and values in $[0,1]$.

Definition 2.2. A binary operation $\square:[0,1] \times[0,1] \square[0,1]$ is called a continuous t-norm if, $([0,1], \square)$ is an abelian topological monoid with unit 1 such that a $\square \mathrm{b} \leq \mathrm{c} \square \square \mathrm{d}$ whenever $\mathrm{a} \leq \mathrm{c}$ and $\mathrm{b} \leq \mathrm{d}$, for all $\mathrm{a}, \mathrm{b}, \mathrm{c}, \mathrm{d}$ in $[0,1]$.

For an example: $\mathrm{a} * \mathrm{~b}=\mathrm{ab}, \mathrm{a} * \mathrm{~b}=\min \{\mathrm{a}, \mathrm{b}\}$.

Definition 2.3 The triplet (X, M, $\square$ ) is called a fuzzy metric space (shortly, a FM-space) if, $\mathrm{X}$ is an arbitrary set, $\square$ is a continuous t-norm and $\mathrm{M}$ is a fuzzy set on $\mathrm{X} \times \mathrm{X} \times[0,1)$ satisfying the following conditions: for all $x, y, z$ in $\mathrm{X}$, and $\mathrm{s}$, $\mathrm{t}>0$,

(i) $\mathrm{M}(x, y, 0)=0, \mathrm{M}(x, y, \mathrm{t})>0$;

(ii) $\mathrm{M}(x, y, \mathrm{t})=1$ for all $\mathrm{t}>0$ if and only if $x=y$,

(iii) $\mathrm{M}(x, y, \mathrm{t})=\mathrm{M}(y, x, \mathrm{t})$,

(iv) $\mathrm{M}(x, y, \mathrm{t}) \square \mathrm{M}(y, z, \mathrm{~s}) \leq \mathrm{M}(x, z, \mathrm{t}+\mathrm{s})$,

(v) $\mathrm{M}(x, y, \cdot):[0, \infty) \square[0,1]$ is left continuous.

In this case, $\mathrm{M}$ is called a fuzzy metric on $\mathrm{X}$ and the function $\mathrm{M}(x, y, \mathrm{t})$ denotes the degree of nearness between $x$ and $y$ with respect to $t$.

Also, we consider the following condition in the fuzzy metric space (X, M, $\square)$ :

(vi) $\lim _{\mathrm{t} \square \text { o }} \mathrm{M}(x, y, \mathrm{t})=1$, for all $x, y \square \square \mathrm{X}$.

It is important to note that every metric space $(\mathrm{X}, \mathrm{d})$ induces a fuzzy metric space (X,M, $\square$ ) where a $\square \mathrm{b}=\min \{\mathrm{a}, \mathrm{b}\}$ and for all a, $\mathrm{b} \square \square \mathrm{X}$,we have $M(x, y, t)=\frac{t}{t+d(x, y)}$, for all $\mathrm{t}>0$, and $\mathrm{M}(x, y, 0)=0$, so-called the fuzzy metric space induced by the metric $\mathrm{d}$.

Definition 2.4. A sequence $\left\{x_{n}\right\}$ in a fuzzy metric space (X, $M, \square$ ) is said to be convergent to $x$ in $X$ if, $\lim _{n \square \infty} M(x n, x$, $\mathrm{t})=1$, for each $\mathrm{t}>0$.

Definition 2.5. A sequence $\left\{x_{n}\right\}$ in a fuzzy metric space (X, $M, \square)$ is called a Cauchy sequence if, $\lim _{n \square \infty} M\left(x_{n+p}, x_{n}, t\right)=$ 1 for every $t>0$ and for each $p>0$. A fuzzy metric space $(\mathrm{X}, \mathrm{M}, \square)$ is complete if, every Cauchy sequence in $\mathrm{X}$ converges in $\mathrm{X}$.

Definition 2.6. Two self mappings $A$ and $S$ of an fuzzy metric space $(X ; M ; *)$ are called compatible if $\lim _{n \square \infty}$ $\mathrm{M}\left(\mathrm{ASx}_{\mathrm{n}} ; \mathrm{SAx}_{\mathrm{n}} ; \mathrm{t}\right)=1$ whenever $\left\{\mathrm{x}_{\mathrm{n}}\right\}$ is a sequence in $\mathrm{X}$ such that $\lim _{\mathrm{n} \square \infty} A x_{\mathrm{n}}=\lim _{\mathrm{n} \square \infty} S \mathrm{x}_{\mathrm{n}}=\mathrm{x}$ for some $\mathrm{x}$ in $\mathrm{X}$.

Definition 2.7. Two self mappings $A$ and $S$ of an fuzzy metric space $\left(\mathrm{X} ; \mathrm{M} ;{ }^{*}\right)$ are called compatible of type $(\mathrm{P})$ if $\lim _{n \square \infty} \mathrm{M}\left(\mathrm{AAx}_{\mathrm{n}} ; \mathrm{SSx}_{\mathrm{n}} ; \mathrm{t}\right)=1$ whenever $\left\{\mathrm{x}_{\mathrm{n}}\right\}$ is a sequence in $\mathrm{X}$ such that $\lim _{\mathrm{n} \square \infty} \mathrm{Ax}_{\mathrm{n}}=\lim _{\mathrm{n} \square \infty} \mathrm{Sx}_{\mathrm{n}}=\mathrm{x}$ for some $\mathrm{x}$ in $\mathrm{X}$. 


\section{International Journal of Science and Research (IJSR) \\ ISSN (Online): 2319-7064}

Index Copernicus Value (2013): 6.14 | Impact Factor (2015): 6.391

\section{Results}

Now, let $\left(\mathrm{X}, \mathrm{M},{ }^{*}\right)$ be a complete fuzzy metric space and let $\mathrm{A}, \mathrm{B}, \mathrm{S}$ and $\mathrm{T}$ be self-mappings of $\mathrm{X}$ satisfying the following conditions; For some positive integers $\mathrm{a}$, $\mathrm{b}$ such that [3.1.1] $\mathrm{A}^{\mathrm{a}}(\mathrm{X}) \subset \mathrm{A}(\mathrm{X}) \subset \mathrm{T}(\mathrm{X})$ and $\mathrm{B}^{\mathrm{b}}(\mathrm{X}) \subset \mathrm{B}(\mathrm{X}) \subset \mathrm{S}(\mathrm{X})$, [3.1.2] $M\left(A^{a} x, B^{b} y, t\right) \geq \varphi\left(\min \left\{M(T x, S y, t), M\left(T x, A^{a} x, t\right), M\left(B^{b}\right.\right.\right.$ $y, S y, t)\}$ ) for all $x, y \in X$, where $\varphi:[0,1] \rightarrow[0,1]$ is a continuous function such that $\varphi(s)>s$ for each $0<s<1$. Then for any $\mathrm{x}_{0}$ be a arbitrary point in $\mathrm{X}$. Since $\mathrm{A}^{\mathrm{a}}(\mathrm{X}) \subset$ $\mathrm{T}(\mathrm{X}), \exists \mathrm{x}_{1}$ in $\mathrm{X}$ such that $\mathrm{Tx}_{1}=\mathrm{A}^{\mathrm{a}} \mathrm{x}_{0}$. Again since $\mathrm{B}^{\mathrm{b}}(\mathrm{X}) \subset$ $\mathrm{S}(\mathrm{X}), \exists \mathrm{X}_{2}$ in $\mathrm{X}$ such that $\mathrm{Sx}_{2}=\mathrm{B}^{\mathrm{b}} \mathrm{x}_{1}$. An general there exists $\mathrm{X}_{2 \mathrm{n}+2}$ in $\mathrm{X}$ such that

[3.1.3] $\mathrm{y}_{2 \mathrm{n}}=\mathrm{Tx}_{2 \mathrm{n}+1}=\mathrm{A}^{\mathrm{a}} \mathrm{x}_{2 \mathrm{n}}$ and $\mathrm{y}_{2 \mathrm{n}+1}=\mathrm{Sx}_{2 \mathrm{n}+2}=\mathrm{B}^{\mathrm{b}} \mathrm{x}_{2 \mathrm{n}+1}$ for $\mathrm{n}$ $=0,1,2, \ldots$

Firstly, we prove the following LEMMA .

LEMMA 4.1. Let A,B, $S$ and $T$ be self-mappings of a fuzzy metric space $\left(\mathrm{X}, \mathrm{M},{ }^{*}\right)$ satisfying the conditions [3.1.1] and [3.1.2]. Then the sequence $\left\{y_{n}\right\}$ defined by [3.1.3] is a Cauchy sequence in $\mathrm{X}$.

PROOF. For $\mathrm{t}>0$,

$M\left(y_{2 n}, y_{2 n+1}, t\right)=M\left(A^{a} x_{2 n}, B^{b} x_{2 n+1}, t\right)$

$\geq \varphi\left(\min \left\{M\left(T_{2 n}, \quad S x_{2 n+1}, t\right), M\left(T x_{2 n}, A^{a} x_{2 n}, t\right), M\left(S x_{2 n+1}\right.\right.\right.$, $\left.\left.\left.\mathrm{B}^{\mathrm{b}} \mathrm{x}_{2 \mathrm{n}+1}, \mathrm{t}\right)\right\}\right)$

$=\varphi\left(\min \left\{M\left(y_{2 n-1}, y_{2 n}, t\right), M\left(y_{2 n-1}, y_{2 n}, t\right), M\left(y_{2 n}, y_{2 n+1}, t\right)\right\}\right)$ [3.1.4]

$> \begin{cases}M\left(y_{2 n-1}, y_{2 n}, t\right), & \text { if } M\left(y_{2 n-1}, y_{2 n}, t\right)<M\left(y_{2 n}, y_{2 n+1}\right. \\ M\left(y_{2 n}, y_{2 n+1}, t\right), & \text { if } M\left(y_{2 n-1}, y_{2 n}, t\right) \geq M\left(y_{2 n}, y_{2 n}\right.\end{cases}$

as $\varphi(s)>s$ for $0<s<1$. Thus $\left\{\mathrm{M}\left(\mathrm{y}_{2 \mathrm{n}}, \mathrm{y}_{2 \mathrm{n}+1}, \mathrm{t}\right), \mathrm{n} \geq 0\right\}$ is an increasing sequence of positive real numbers in $[0,1]$ and therefore tends to a limit $l \leq 1$. We assert that $l=1$. If not, $l$ $<1$ which on letting $n \rightarrow \infty$ in [3.1.4] one gets $l \geq \varphi(1)>l$ a contradiction yielding thereby $\mathrm{l}=1$. Therefore for every $n \in$ $\mathrm{N}$, using an alogous arguments one can show that $\left\{\mathrm{M}\left(\mathrm{y}_{2 \mathrm{n}+1}\right.\right.$, $\left.\left.y_{2 n+2}, t\right), n \geq 0\right\}$ is a sequence of positive real numbers in $[0$, $1]$ which tends to a limit $l=1$. Therefore for every $n \in N$

$M\left(y_{n}, y_{n+1}, t\right)>M\left(y_{n-1}, y_{n}, t\right)$ and $\lim _{n \rightarrow \infty} M\left(y_{n}, y_{n+1}, t\right)=1$.

Now for any positive integer $\mathrm{p}$

$\mathrm{M}\left(\mathrm{y}_{\mathrm{n}}, \mathrm{y}_{\mathrm{n}+\mathrm{p}}, \mathrm{t}\right) \geq \mathrm{M}\left(\mathrm{y}_{\mathrm{n}}, \mathrm{y}_{\mathrm{n}+1}, \mathrm{t} / \mathrm{p}\right) * \ldots * \mathrm{M}\left(\mathrm{y}_{\mathrm{n}+\mathrm{p}-1}, \mathrm{y}_{\mathrm{n}+\mathrm{p}}, \mathrm{t} / \mathrm{p}\right)$.

Since $\lim _{n \rightarrow \infty} M\left(y_{n}, y_{n+1}, t\right)=1$ for $t>0$, it follows that

$\lim _{\mathrm{n} \rightarrow \infty} \mathrm{M}\left(\mathrm{y}_{\mathrm{n}}, \mathrm{y}_{\mathrm{n}+\mathrm{p}}, \mathrm{t}\right) \geq 1 * 1 * \ldots * 1=1$

which shows that $\left\{y_{n}\right\}$ is a Cauchy sequence in $X$.

Now we prove our main result as follows:

Theorem: Let A,B, S and $\mathrm{T}$ be four self-mappings of a fuzzy metric space (X,M, *) satisfying the condition [3.1.2]. if [3.1.1] and one of $\mathrm{A}(\mathrm{X}), \mathrm{B}(\mathrm{X}), \mathrm{S}(\mathrm{X})$ and $\mathrm{T}(\mathrm{X})$ is a complete subspace of $X$, then if the pairs $\left\{T, A^{a}\right\}$ and $\{S$, $\left.\mathrm{B}^{\mathrm{b}}\right\}$ are compatible mapping of type $(\mathrm{P})$, then $\mathrm{A}, \mathrm{B}, \mathrm{S}$ and $\mathrm{T}$ have a unique common fixed point.

Proof: Let $\mathrm{x}_{0}$ be an arbitrary point in $\mathrm{X}$. Then following arguments of Fishe [7], one can construct sequences $\left\{x_{n}\right\}$ and $\left\{\mathrm{y}_{\mathrm{n}}\right\}$ in $\mathrm{X}$ such that $\mathrm{y}_{2 \mathrm{n}}=\mathrm{Tx}_{2 \mathrm{n}+1}=\mathrm{A}^{\mathrm{a}} \mathrm{x}_{2 \mathrm{n}}$ and $\mathrm{y}_{2 \mathrm{n}+1}=\mathrm{Sx}_{2 \mathrm{n}+2}=\mathrm{B}^{\mathrm{b}} \mathrm{x}_{2 \mathrm{n}+1}$ for $\mathrm{n}=0,1,2$,

...

Then due to LEMMA [4.1], suppose that $S(X)$ is a complete subspace of $X$, then the subsequence $\mathrm{y}_{2 \mathrm{n}+1}=\mathrm{Sx}_{2 \mathrm{n}+2}$ must get a limit in $\mathrm{S}(\mathrm{X})$. As $\left\{\mathrm{y}_{\mathrm{n}}\right\}$ is a Cauchy sequence containing a convergent subsequence $\left\{y_{2 n+1}\right\}$, therefore the sequence $\left\{y_{n}\right\}$ also converges implying thereby the convergence of $\left\{y_{2 n}\right\}$ being a subsequence of the convergent sequence $\left\{y_{n}\right\}$. Since $\left\{\mathrm{A}^{\mathrm{a}} \mathrm{x}_{2 \mathrm{n}}\right\}$ and $\left\{\mathrm{B}^{\mathrm{b}} \mathrm{x}_{2 \mathrm{n}+1}\right\}$ are subspace of $\left\{\mathrm{y}_{\mathrm{n}}\right\}$ so these subsequence are also converges to a point $\mathrm{w}$. they also converge to the same limit $\mathrm{w}$. Hence there exist two points $\mathrm{u}, \mathrm{v}$ in $\mathrm{X}$ such that $\mathrm{Tu}=\mathrm{w}$ and $\mathrm{Sv}=\mathrm{w}$, respectively. On setting $x=v$ and $y=x_{2 n+1}$ in [3.1.2] we get

$M\left(A^{a} u, y_{2 n+1}, t\right)=M\left(A^{a} u, B^{b} x_{2 n+1}, t\right)$

$\geq \varphi\left(\min \left\{M\left(T u, S x_{2 n+1}, t\right), M\left(T u, A^{a} u, t\right), M\left(S x_{2 n+1}, B^{b} x_{2 n+1}\right.\right.\right.$, t) $\})$

$\geq \varphi\left(\min \left\{M\left(T u, y_{2 n}, t\right), M\left(T u, A^{a} u, t\right), M\left(y_{2 n}, y_{2 n+1}, t\right)\right\}\right)$

$M\left(A^{a} u, w, t\right) \geq \varphi\left(\min \left\{M(T u, w, t), M\left(T u, A^{a} u, t\right), M(w, w\right.\right.$, t)\})

$\mathrm{M}\left(\mathrm{A}^{\mathrm{a}} \mathrm{u}, \mathrm{w}, \mathrm{t}\right) \geq \varphi\left(\min \left\{\mathrm{M}(\mathrm{w}, \mathrm{w}, \mathrm{t}), \mathrm{M}\left(\mathrm{w}, \mathrm{A}^{\mathrm{a}} \mathrm{u}, \mathrm{t}\right), \mathrm{M}(\mathrm{w}, \mathrm{w}, \mathrm{t})\right\}\right)$

$M\left(A^{a} u, w, t\right) \geq \varphi\left(\min \left\{M\left(w, A^{a} u, t\right),\right\}\right)$

$\mathrm{M}\left(\mathrm{A}^{\mathrm{a}} \mathrm{u}, \mathrm{w}, \mathrm{t}\right) \geq \varphi\left(\mathrm{M}\left(\mathrm{A}^{\mathrm{a}} \mathrm{u}, \mathrm{w}, \mathrm{t}\right)\right)>\mathrm{M}\left(\mathrm{A}^{\mathrm{a}} \mathrm{u}, \mathrm{w}, \mathrm{t}\right)$

A contradiction. Therefore $A^{a} u=w=T u$, that is $u$ is a coincidence of $\mathrm{A}^{\mathrm{a}}$ and $\mathrm{T}$.

Similarly, we can show that $\mathrm{v}$ is a coincidence point of $\mathrm{B}^{\mathrm{b}}$ ateld S.

Thinere exist two points $\mathrm{u}, \mathrm{v}$ in $\mathrm{X}$ such that $\mathrm{A}^{\mathrm{a}} \mathrm{u}=\mathrm{Tu}=\mathrm{w}$ and $\mathrm{B}^{\mathrm{b}} \mathrm{v}=\mathrm{Sv}=\mathrm{W}$, respectively, then $\left\{\mathrm{T}, \mathrm{A}^{\mathrm{a}}\right\}$ and $\left\{\mathrm{S}, \mathrm{B}^{\mathrm{b}}\right\}$ is also compatible mapping of type(P).

[3.1.5] $\mathrm{A}^{\mathrm{a}} \mathrm{A}^{\mathrm{a}} \mathrm{x}_{2 \mathrm{n}}=\mathrm{TTX}_{2 \mathrm{n}} \rightarrow \mathrm{Tw}$

$\mathrm{B}^{\mathrm{b}} \mathrm{B}^{\mathrm{b}} \mathrm{x}_{2 \mathrm{n}+1}=\mathrm{SSx}_{2 \mathrm{n}+1} \rightarrow \mathrm{Sw}$

which implies that $A^{a} w=T w$, and $B^{b} w=S w$. Now, we prove that $A^{\mathrm{a}} \mathrm{W}=\mathrm{w}$. If $\mathrm{A}^{\mathrm{a}} \mathrm{w} \neq \mathrm{w}$, then by [3.1.2], we have

$M\left(A^{a} w, y_{2 n+1}, t\right)=M\left(A^{a} w, B^{b} x_{2 n+1}, t\right)$

$\geq \varphi\left(\min \left\{\mathrm{M}\left(\mathrm{Tw}, \mathrm{Sx}_{2 \mathrm{n}+1}, \mathrm{t}\right), \mathrm{M}\left(\mathrm{Tw}, \mathrm{A}^{\mathrm{a}} \mathrm{w}, \mathrm{t}\right), \mathrm{M}\left(\mathrm{Sx}_{2 \mathrm{n}+1}\right.\right.\right.$, $\left.\left.\left.\mathrm{B}^{\mathrm{b}} \mathrm{x}_{2 \mathrm{n}+1}, \mathrm{t}\right)\right\}\right)$

$\geq \varphi\left(\min \left\{M\left(T w, y_{2 n}, t\right), M\left(T w, A^{a} w, t\right), M\left(y_{2 n}, y_{2 n+1}, t\right)\right\}\right)$

$M\left(A^{a} w, w, t\right) \geq \varphi\left(\min \left\{M(T w, w, t), M\left(T w, A^{a} w, t\right), M(w\right.\right.$, $\mathrm{w}, \mathrm{t})\})$

$M\left(A^{a} w, w, t\right) \geq \varphi\left(\min \left\{M(w, w, t), M\left(w, A^{a} u, t\right), M(w, w\right.\right.$, t) $\})$

$M\left(A^{a} w, w, t\right) \geq \varphi\left(\min \left\{M\left(w, A^{a} w, t\right),\right\}\right)$

$\mathrm{M}\left(\mathrm{A}^{\mathrm{a}} \mathrm{w}, \mathrm{w}, \mathrm{t}\right) \geq \varphi\left(\mathrm{M}\left(\mathrm{A}^{\mathrm{a}} \mathrm{w}, \mathrm{w}, \mathrm{t}\right)\right)>\mathrm{M}\left(\mathrm{A}^{\mathrm{a}} \mathrm{w}, \mathrm{w}, \mathrm{t}\right)$

a contradiction. Hence $A^{a} w=w=T w$. Similarly, we have $\mathrm{B}^{\mathrm{b}} \mathrm{w}=\mathrm{Sw}=\mathrm{w}$.

This means that $\mathrm{w}$ is a common fixed point of $\mathrm{A}^{\mathrm{a}}, \mathrm{B}^{\mathrm{b}}, \mathrm{T}$ and S.

Hence $\mathrm{Az}=\mathrm{Tz}=\mathrm{z}=\mathrm{Bz}=\mathrm{Sz}$.

This complete the proof. 


\section{International Journal of Science and Research (IJSR) \\ ISSN (Online): 2319-7064}

Index Copernicus Value (2013): 6.14 | Impact Factor (2015): 6.391

\section{For Uniqueness of Common Fixed Point}

Suppose $\mathrm{z}$ and $\mathrm{w}$ be two distinct common fixed point of A, $B, T$ and $S$. Then from [3.1.2], we have

$M(w, z, t)=M\left(A^{a} w, B^{b} z, t\right) \geq \varphi(\min \{M(T w, S z, t), M(T w$, $\left.\left.\left.A^{\mathrm{a}} w, t\right), M\left(S z, B^{b} z, t\right)\right\}\right)$

$\geq \varphi(\min \{\mathrm{M}(\mathrm{w}, \mathrm{z}, \mathrm{t}), \mathrm{M}(\mathrm{w}, \mathrm{w}, \mathrm{t}), \mathrm{M}(\mathrm{z}, \mathrm{z}, \mathrm{t})\})$

$\mathrm{M}(\mathrm{w}, \mathrm{z}, \mathrm{t}) \geq \varphi(\min \{\mathrm{M}(\mathrm{w}, \mathrm{z}, \mathrm{t})\})>\min \{\mathrm{M}(\mathrm{w}, \mathrm{z}, \mathrm{t})\}$

which is contradiction. Hence $\mathrm{z}$ is unique common fixed point of A, B, T and S.

\section{References}

[1] D.W. Boyd, J. S.Wong, On nonlinear contractions, Proc. Amer. Math. Soc. 20(1969), 458-462.

[2] Y. J.Cho, Fixed points in fuzzy metric spaces, J. Fuzzy Math. 5(1997), 949- 962.

[3] R. Chugh, S. Kumar, Common fixed point theorem in fuzzy metric spaces, Bull. Calcutta Math. Soc. 94,(2002) 17-22.

[4] Z. Deng, Fuzzy pseudo-metric space, J. Math. Anal. Appl. 86(1982), 74-95.

[5] M.A. Erceg, Metric space in fuzzy set theory, J. Math. Anal. Appl. 69(1979), 205-230.

[6] J. X. Fang, On fixed point theorems in fuzzy metric spaces, Fuzzy Sets Systems 46(1992), 107-113.

[7] B. Fisher, Common fixed points of four mappings, Bull. Inst. Math. Acad. Sinica 11(1983), 103-113.

[8] A. George, P. Veeramani, On some results in fuzzy metric spaces, Fuzzy Sets Systems 64(1994), 395-399.

[9] A. George, P.Veeramani, On some results of analysis for fuzzy metric spaces, Fuzzy Sets Systems 90(1997), 365-368.

[10] M. Grabiec, Fixed points in fuzzy metric space, Fuzzy Sets Systems 27(1988), 385-389.

[11] O. Kaleva, S. Seikkala, On fuzzy metric spaces, Fuzzy Sets Systems 12(1984), 215-229.

[12] I. Kramosil, J.Michalek, Fuzzy metric and statistical metric spaces, Kybernetica 15(1975), 326-334.

[13] R. P. Pant, Common fixed point for non commuting mappings, J. Math. Anal. Appl. 188(1994), 436-440.

[14] R. P.Pant, K. Jha, A remark on common fixed points of four mappings in a fuzzy metric space, J. Fuzzy Math. 12(2004), 433-437.

[15] H. K. Pathak, Y. J.Cho, S.M.Kang, Remarks on Rweakly commuting mappings and common fixed point theorems, Bull. Korean Math. Soc. 34(1997), 247-257.

[16] B. Schweizer, A. Sklar, Statistical metric spaces, Pacific J. Math. 10(1960), 313-334.

[17] G. Song, Comments on a common fixed point theorem in fuzzy metric space, Fuzzy Sets Systems 135(2003), 409-413.

[18] R.Vasuki, Common fixed points for R-weakly commuting maps in fuzzy metric spaces, Indian J. Pure Appl. Math. 30(1999), 419-423.

[19] R.Vasuki, P.Veeramani, Fixed point theorems and Cauchy sequences in fuzzy metric spaces, Fuzzy Sets Systems 135(2003), 415-417.

[20] L. A. Zadeh, Fuzzy sets, Inform. Control 8(1965), 338353.

\section{Author Profile}

Raghu Nandan Patel Department of Mathematics, Government Mukut Dhar Pandey College, Katghora Distt - Korba Chhattisgarh, India

Manoj Kumar Tiwari Department of Mathematics, Government Girls Polytechnic, Bilaspur, Chhattisgarh, India 